\title{
Nonlinear Operations for Colour Images Based on Pairwise Vector Ordering
}

\author{
Adrian N. Evans \\ Department of Electronic and Electrical Engineering \\ University of Bath \\ Bath, BA2 7AY \\ United Kingdom \\ A.N.Evans@bath.ac.uk
}

\begin{abstract}
A new vector ordering is proposed and its application to colour image processing demonstrated. Partial ordering is first used rank the vectors in groups of two and then a simple reduced ordering is applied to each pair to give a full ordering. As the initial ordering is based on pairs of vectors, the technique is termed pairwise vector ordering. The new ordering is used to develop a median filter and colour morphological operations. A quantitative evaluation shows the pairwise vector median to be less effective for noise reduction than the well known vector median filter, albeit with reduced complexity. Colour morphological erosions and dilations based on the pairwise ordering are shown to produce well behaved results that conform with expectations, without any of the problems associated with applying the operations to the individual channels. Their application to multiscale image analysis is also investigated.
\end{abstract}

\section{Introduction}

A large number nonlinear filters have been developed for the processing of greyscale images. Many of these, such as the median filter and the erosion and dilation operations of mathematical morphology, require the pixels contained within a mask or structuring element to be ordered. Therefore, as no unambiguous ordering for vector data exists, their extension to colour and other multivariate images is not straightforward. In particular, the scalar median, minimum and maximum operations do not have unique multivariate equivalents and instead a number of vector ordering schemes have been proposed. In his classic paper, Barnett categorises sub-ordering schemes for multivariate data as marginal ordering, reduced (or aggregate) ordering, partial ordering and conditional ordering [2].

In marginal ordering (M-ordering), the scalar ranking is performed within one or more of the individual channels.
For colour imagery, this has the disadvantage of generating colours that were not present in the original samples. Reduced ordering (R-ordering) reduces each multivariate sample to a scalar value that is a function of the observations in each channel and has been widely used in colour image processing. Lexicographic ordering, which orders data according to a hierarchy of channels, is the best known example of conditional ordering (C-ordering) and has found application in colour morphology [10]. Finally, partial ordering (P-ordering) partitions the data into groups that are ranked with respect to each other. The operation is normally based on the convex hull and so is computationally expensive and, furthermore, no intra-group ranking exists.

In this paper, a new approach to vector ordering is presented and examples of its use in nonlinear operations for colour images given. The technique is termed pairwise vector ordering as it first orders vectors in groups of two, according to scalar distances between each pair of vectors. After all pairs have been ranked, for an odd number of samples the median vector is defined as the remaining unpaired vector and is therefore one of the original set. The highest ranked pair of vectors contains the maximum and minimum vectors and a simple reduced ordering can be applied to identify which is which. A complete ordering can then be achieved by applying the reduced ordering to each vector pair. As such, the technique is a combination of $\mathrm{P}$-ordering and R-ordering. Colour filters based on various vector ordering schemes are briefly reviewed in section 2 and the new ordering scheme described in section 3 . Results and discussion are presented in section 4 and conclusions drawn in section 5 .

\section{Vector ordering for multichannel median and morphological operations}

Vector order statistics employing R-ordering have been used in the design of many multichannel filters including 
the vector median [1] and colour edge detectors [16, 18]. For a set of $n$ vectors, $\vec{x}_{1}, \vec{x}_{2}, \cdots \vec{x}_{n}$, the aggregate distance of $\vec{x}_{i}$ to the other vectors in the set is denoted $d_{i}$ and given by

$$
d_{i}=\sum_{k=1}^{n}\left\|\vec{x}_{i}-\vec{x}_{k}\right\|_{p}, \quad i=1,2, \cdots, n,
$$

where $\|\cdot\|_{p}$ is a vector norm. When the vectors are ordered according to their $d_{i} s$ such that $d_{(1)} \leq d_{(2)} \leq \cdots \leq d_{(n)}$ the result is the ordered sequence

$$
\vec{x}_{(1)} \leq \vec{x}_{(2)} \leq \cdots \leq \vec{x}_{(n)} .
$$

With this ordering, $\vec{x}_{(1)}$ is the vector median, whose aggregate distance to the other vectors is less than or equal to the aggregate distances of all other vectors; this vector is employed by the well known vector median filter of [1]. An alternative to using aggregate distances based on norms is to consider the vectors' directions and the approach taken by vector directional filter is to order vectors according to the aggregate difference in angles [17]. More recently, vector filters that combine the advantages of both direction and magnitude have been developed [12].

Whatever distance measure is used for the aggregate distance, $\vec{x}_{(n)}$ is the vector extremum. However, unlike the scalar case where two extrema (the maximum and the minimum) exist, the multivariate ordering of equations 1 and 2 only defines a single extremum, given by the highest ranked vector. This is easily understood by applying the single channel form of the equations to three scalar values, when the value with the highest rank is the one that is furthest from the median; this value could be the scalar maximum or minimum. Therefore, although vector order statistics provide a good theoretical basis for defining the vector median, they cannot easily be employed for extending mathematical morphological operations to multichannel images, where the vector maximum and vector minimum are required. This problem has been overcome in morphology sieves based on graph morphology where the opening and closing operations are implemented by merging regional extrema with their closest neighbours. Using this approach two colour sieves have been proposed $[4,7]$ and evaluated $[8,9]$.

The problem of extending structuring element-based morphological operations from univariate to multivariate images has also received some attention. Comer and Delp propose vector dilation and erosion operators for colour image processing using both M-ordering and R-ordering [3]. Operations employing M-ordering simply apply standard morphological operations to each channel. Although Mordering can be implemented in any colour space, it can modify the spectral composition of the image and produce jitter near edges. R-ordering effectively selects one of the input vectors as the output and so does not produce colours

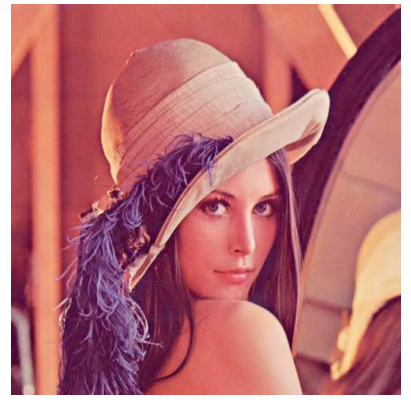

(a)

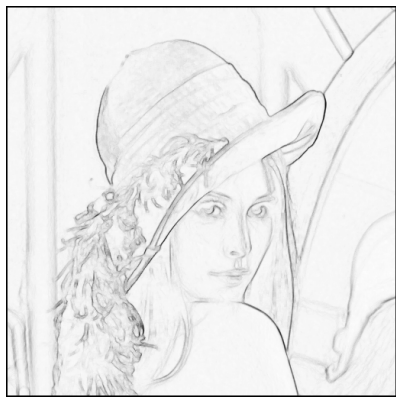

(b)

\section{Figure 1. Robust colour morphological exam- ple using a $5 \times 5$ mask and distance $d_{8}$ in equa- tion 4. (a) Lenna test image and (b) gradient magnitude.}

that were not present in the original image. Suitable scalar functions that were investigated include the luminance and individual colour channels [3]. Other authors have proposed a combination of reduced and conditional ordering [14], marginal ordering [15] and lexicographical ordering in the HLS colour space [10]. More recently, Li and Li introduced a R-ordering scheme based on principal component analysis [11].

\section{Pairwise vector ordering}

To overcome the difficulty the R-ordering scheme described above has in defining the minimum and maximum vectors, a new vector ordering scheme is proposed. The scheme first uses P-ordering to rank vectors in groups of 2. As the P-ordering is based on vector differences rather than the convex hull it is relatively efficient. R-ordering is then applied to each pair of vectors to induce a full ordering. The approach is therefore a combination of P-ordering and $\mathrm{R}$-ordering and is termed pairwise ordering as it is based on groups of 2 vectors.

The first stage in the pairwise vector ordering scheme is to find the scalar distance

$$
d_{0}=\max _{i, j \in\{1,2, \cdots, n\}}\left(\left\|\vec{x}_{i}-\vec{x}_{j}\right\|_{p}\right) .
$$

This is the maximum distance between any two of the $n$ vectors and the two vectors associated with $d_{0}$ are the maximum and minimum vectors, although it is not known which is which. If these two vectors are removed and the maximum distance found again, $d_{1}$ results. Repeating this operation results in an ordered set of distances $d_{0} \geq d_{1} \geq \cdots \geq$ $d_{(n / 2)-1}$ that are given by the vectors norms 


$$
\begin{aligned}
\left\|\vec{x}_{(1)}-\vec{x}_{(n)}\right\|_{p} & \geq\left\|\vec{x}_{(2)}-\vec{x}_{(n-1)}\right\|_{p} \geq \\
\cdots & \geq\left\|\vec{x}_{\left(\frac{n}{2}\right)}-\vec{x}_{\left(\frac{n}{2}+1\right)}\right\|_{p},
\end{aligned}
$$

where $\vec{x}_{(s+1)}$ and $\vec{x}_{(n-s)}$ are the two vectors associated with distance $d_{s}$ and are interchangeable. This approach therefore orders the vectors in pairs.

The distances in equation 4 have been successfully used in colour edge detection, where $d_{0}$ and $d_{s}$ for $s>0$ form the basis of the colour morphological gradient (CMG) and the robust colour morphological gradient (RCMG) respectively $[5,6]$. For edge detection, it is the difference between the two vectors in each pair that is important and so there is no requirement to rank the vectors within each group. An example of the gradient magnitude for the Lenna test image is shown in figure 1 and for a detailed description and evaluation of the RCMG operator the reader is referred to [6].

The pairwise ranking of equation 4 can be used to order $n / 2$ vector pairs when $n$ is even. When $n$ is odd one unpaired vector remains after the other $n-1$ vectors are ordered. This vector (denoted $\vec{x}_{m}$ ) is assigned the distance $d_{s}=\left\|\vec{x}_{m}-\vec{x}_{m}\right\|_{p}=0$, which is the lowest ranked distance. In a manner reminiscent of the vector median filter, the vector associated with the lowest ranked distance can be uniquely identified as the median vector. When $n$ is even, there are two vectors associated with the lowest ranked position, both of which are candidates for the median vector. This situation is identical to that encountered when finding the scalar median of an even number of samples where the usual, if somewhat unsatisfactory, approach is to define the median as the mean of the two central samples.

To move from a pairwise to a complete vector ranking a mechanism for ordering the pair of vectors within each group is needed. The method proposed here is based on the observation when equation 4 is applied to univariate data the two values associated with distance $d_{0}$ are the maximum and minimum. Taking the approach that, for each pair of vectors, the maximum (resp. minimum) vector has a greatest (resp. smallest) magnitude, a complete vector ordering is given by

$$
\vec{x}_{(1)} \leq \vec{x}_{(2)} \leq \cdots \leq \vec{x}_{(n-1)} \leq \vec{x}_{(n)}
$$

where $\vec{x}_{(s+1)}$ and $\vec{x}_{(n-s)}$ are the two vectors that give rise to distance $d_{s}$ and $\left\|\vec{x}_{(s+1)}\right\|_{p} \leq\left\|\vec{x}_{(n-s)}\right\|_{p}$. For RGB colour images with values in the range $0-255$ this ordering will result in vectors with a low luminance being given a low rank.

Figure 2 illustrates the operation of the full pairwise ordering scheme for a set of 9 vectors. In this example, the vectors are first ranked into 4 pairs using the Euclidean distance (shown with dotted lines) and one unpaired vector

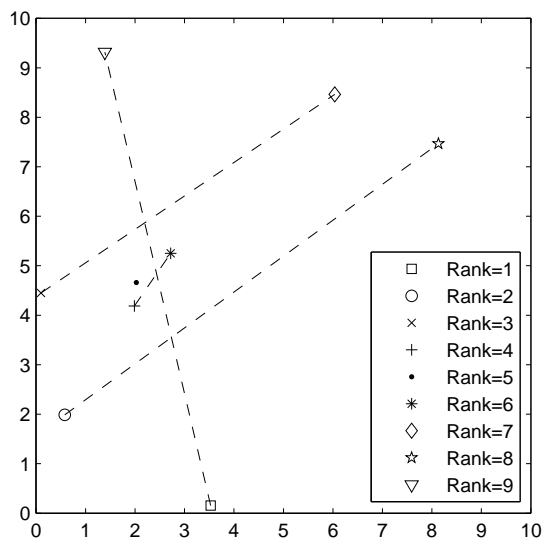

Figure 2. Pairwise ordering example for $n=9$. Distances $d_{s}, s=0,1, \cdots, 3$ shown with dotted lines and complete ordering found using the $L_{2}$ norm.

which is ranked in position $(n+1) / 2=5$. The maximum and minimum vectors in each pair are then determined using the $L_{2}$ norm giving the complete ordering shown in the figure's legend. This example illustrates that full pairwise ordering corresponds well with an intuitive ranking of the vectors: vectors ranked in positions 1 and 9 are the maximum and minimum respectively while mid-ranked vectors (ranks 4-6) provide a good measure of central tendency. The figure also illustrates the role played by the initial pairwise ordering in the vector ranking, producing a ranking that is subtly different from simply ordering by luminance. For example, when the vectors $\vec{x}_{(1)}, \vec{x}_{(2)}, \cdots, \vec{x}_{(9)}$ from figure 2 are ordered by their luminance their ranked positions are 2 , $1,3,4,5,6,9,7$ and 8 .

Using the ordering of equation 5, the morphological operations of colour dilation $\vec{\delta}_{B}$, erosion $\vec{\epsilon}_{B}$, opening $\vec{O}_{B}$ and closing $\vec{C}_{B}$ for colour and other multichannel images can be defined by

$$
\begin{aligned}
\vec{\delta}_{B} & =\max _{i \in B}\left(\vec{x}_{i}\right) \\
\vec{\epsilon}_{B} & =\min _{i \in B}\left(\vec{x}_{i}\right) \\
\vec{O}_{B} & =\vec{\delta}_{B}\left(\vec{\epsilon}_{B}\right) \\
\vec{C}_{B} & =\vec{\epsilon}_{B}\left(\vec{\delta}_{B}\right)
\end{aligned}
$$

respectively, where $B$ is a structuring element.

\section{Evaluation and Discussion}

The first aspect of the pairwise ordering scheme to be considered is its performance as a median filter. For comparison the vector median filter of [1] is used. Figure 3 


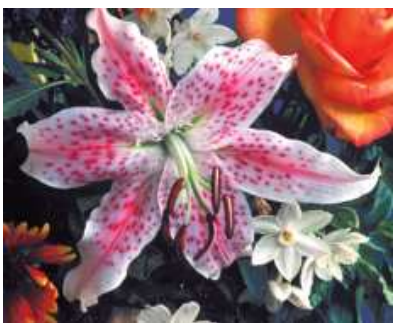

(a) Original image

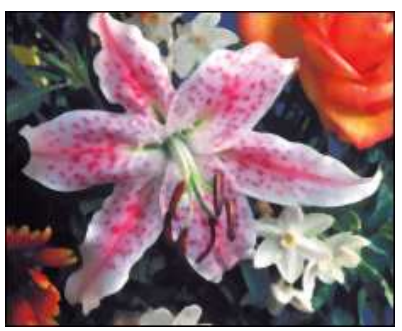

(c) Vector median

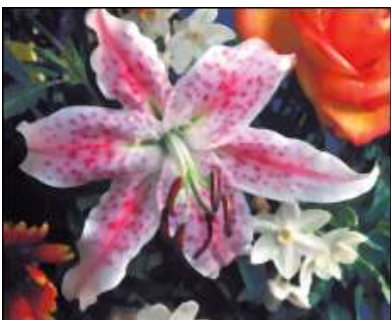

(b) Pairwise median

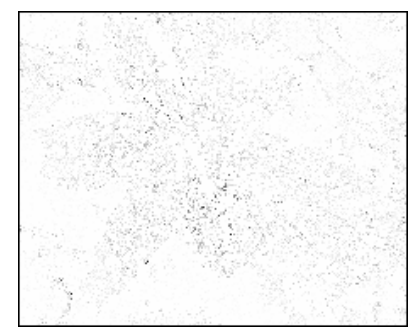

(d) Scaled difference between (b) and (c)

Figure 3. Colour median filter results for Lily image using $3 \times 3$ mask.

presents the results of median filtering the $186 \times 230$ colour test image Lily using a $3 \times 3$ mask. Visually the vector median and pairwise median images (figures 3(b) and (c) respectively) are very similar. However, examination of the difference image in figure 3(d) shows that differences do exist, particularly in some regions of high gradient.

To quantify the amount of image distortion introduced by the filters, the filtered images can be compared to the original using an error metric. Here, the normalised mean square error (NMSE) is used, given by

$$
N M S E=\frac{\sum_{i=0}^{N_{1}} \sum_{j=0}^{N_{2}}\left\|\vec{f}(i, j)-\overrightarrow{f^{\prime}}(i, j)\right\|^{2}}{\sum_{i=0}^{N_{1}} \sum_{j=0}^{N_{2}}\|\vec{f}(i, j)\|^{2}}
$$

where $\vec{f}(i, j)$ and $\vec{f}^{\prime}(i, j)$ are the original and filtered vectors at pixel $(i, j)$ and $N_{1}$ and $N_{2}$ are the image dimensions [17].

Table 1 shows that although the NMSE between the vector and pairwise median filtered images is very low (0.00042), when compared to the original image the vector median filter introduces less slightly image distortion.

\subsection{Noise reduction}

Multichannel median filters have been widely used for reducing noise in colour images. To evaluate the performance of a median filter based on the pairwise ordering,

\begin{tabular}{||l|c|c||}
\hline \hline & $\begin{array}{c}\text { Vector } \\
\text { Median }\end{array}$ & $\begin{array}{c}\text { Pairwise } \\
\text { Median }\end{array}$ \\
\hline \hline Original image & 0.5734 & 0.5824 \\
\hline Vector median & 0 & 0.0418 \\
\hline \hline
\end{tabular}

\section{Table 1. NMSE $\left(\times 10^{-2}\right)$ between Lily and me- dian filtered images using a $3 \times 3$ mask.}

the Lily image was corrupted by independent and correlated noise and the quality of the images after filtering assessed using the NMSE.

To provide a realistic simulation of image noise both Gaussian and impulsive noise models were used. For impulsive noise a simplified version of the noise model described in [19] was employed, in which the proportion of noise applied to each individual channel or all channels simultaneously can be controlled. For a colour pixel $\vec{x}=\left(x_{1}, x_{2}, x_{3}\right)^{T}$ the noise model used was

$$
\vec{x}_{n}= \begin{cases}\vec{x}, & \text { with probability } 1-p_{I} \\ \left(I, x_{2}, x_{3}\right)^{T}, & \text { with probability } p_{I} p_{c} / 3 \\ \left(x_{1}, I, x_{3}\right)^{T}, & \text { with probability } p_{I} p_{c} / 3 \\ \left(x_{1}, x_{2}, I\right)^{T}, & \text { with probability } p_{i} p_{c} / 3 \\ (I, I, I)^{T}, & \text { with probability } p_{I} p_{c},\end{cases}
$$

where $\vec{x}_{n}$ is the noisy pixel, $p_{I}$ is the probability of an impulse of value $I$ and $p_{c}$ is the proportion of correlated noise.

Correlated Gaussian noise was simulated by adding a random vector to each colour pixel $\vec{x}$. The random vectors were selected from the multivariate normal distribution with covariance matrix

$$
\sum=\sigma^{2}\left[\begin{array}{lll}
1 & \rho & \rho \\
\rho & 1 & \rho \\
\rho & \rho & 1
\end{array}\right]
$$

where $\sigma^{2}$ is the variance of the Gaussian distribution and $\rho$ is the correlation coefficient between each pair of channels.

The noise parameters and NMSE results are given in table 2, again using the vector median for comparison. The results show that although the pairwise median filter successfully reduces noise, its NMSE performance is slightly worse than the vector median filter for all noise types. This is perhaps to be expected as the vector median filter uses aggregate distances to define the median while in the pairwise ordering each distance $d_{s}$ only depends on two vectors. It is also noted that developments such as weighted vector filters, 


\begin{tabular}{|c||c|c|c|}
\hline \hline $\begin{array}{c}\text { Noise Model } \\
\text { Model }\end{array}$ & $\begin{array}{c}\text { No } \\
\text { Filter }\end{array}$ & $\begin{array}{c}\text { Pairwise } \\
\text { Median }\end{array}$ & $\begin{array}{c}\text { Vector } \\
\text { Median }\end{array}$ \\
\hline \hline $\begin{array}{c}\text { Independent Gaussian } \\
(\sigma=30)\end{array}$ & 4.7803 & 2.5447 & 2.2586 \\
\hline $\begin{array}{c}\text { Correlated Gaussian } \\
(\sigma=30, \rho=0.5)\end{array}$ & 4.8146 & 2.3309 & 2.1298 \\
\hline $\begin{array}{c}\text { Independent Impulsive } \\
(5 \%)\end{array}$ & 6.3902 & 0.8313 & 0.7736 \\
\hline $\begin{array}{c}\text { Correlated Impulsive } \\
(5 \%, \rho=0.5)\end{array}$ & 4.1479 & 0.6818 & 0.6668 \\
\hline $\begin{array}{c}\text { Correlated Mixed } \\
\text { Gaussian }(\sigma=20) \text { and } \\
\text { impulsive (3\%) }\end{array}$ & 4.6819 & 1.5958 & 1.4778 \\
\hline \hline
\end{tabular}

Table 2. NMSE $\left(\times 10^{-2}\right)$ for colour median filtered images for Lily test image using a $3 \times 3$ mask.

often employing fuzzy rules, out-perform the basic vector median filter [13]. Therefore the use of the pairwise median filter may perhaps be restricted to applications where a reasonable visual appearance is desired with a relatively low computational cost, and without the artifacts associated with processing channels individually.

\subsection{Colour morphology operations}

The basic colour morphological operations based on the pairwise ordering given in equations 6-9 are illustrated in application to the Mandrill test image. Figure 4 shows the original image and the results of colour dilation, erosion, opening and closing using a square $3 \times 3$ structuring element. The results show the performance of the colour morphological operations is consistent with expectations, based on an intuitive understanding of greyscale morphology. For example, the results of erosion and dilation in the eye region show that the appropriate image components are removed, illustrating the potential benefits of these operations. Figure 4 also shows the absence of any problems associated with filtering each channel independently, such as edge jitter.

Applying a series of openings and closings of increasing scale results in a scale-space image representation that can be used for applications such as image compression and texture analysis based on granulometries. The results of opening and closing the Mandrill image with square structuring elements in figure 5 show the rapid simplification of the im-

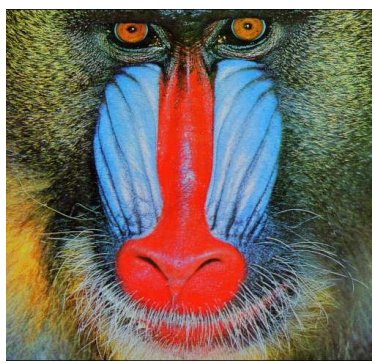

(a) Original image

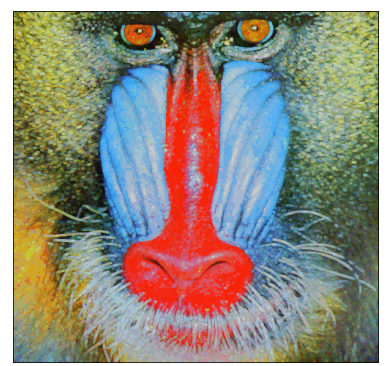

(b) Colour dilation

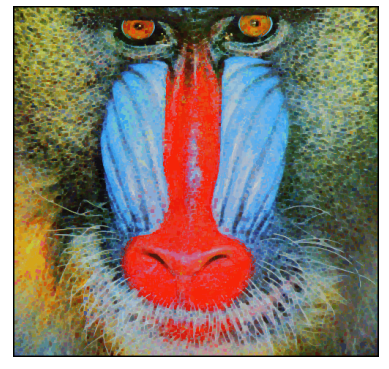

(d) Colour closing

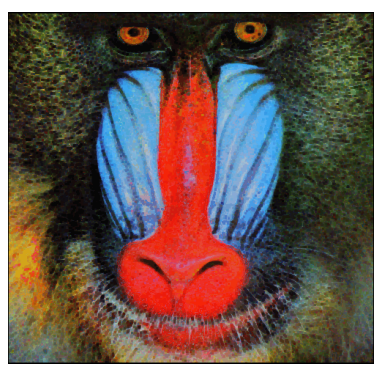

(c) Colour erosion

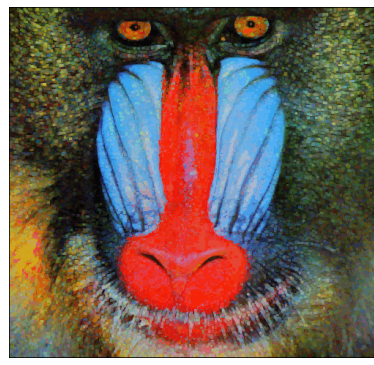

(e) Colour opening
Figure 4. Colour morphological operations applied to Mandrill image using a square $3 \times 3$ structuring element.

age with increasing scale and provide a basis for characterising images based on scale, shape and colour.

\section{Conclusions}

A novel vector ordering scheme termed pairwise ordering has been presented and evaluated. Its ordering is a combination of $\mathrm{P}$ - and R-ordering and this has the advantage of enabling the maximum and minimum vectors to be identified, in addition to the median vector. The ordering is also relatively computationally efficient.

A median filter based on the new ordering was shown to reduce noise but to a lesser degree then the vector median filter. However, unlike the ordering of the vector median, the pairwise ordering also allows the development of 

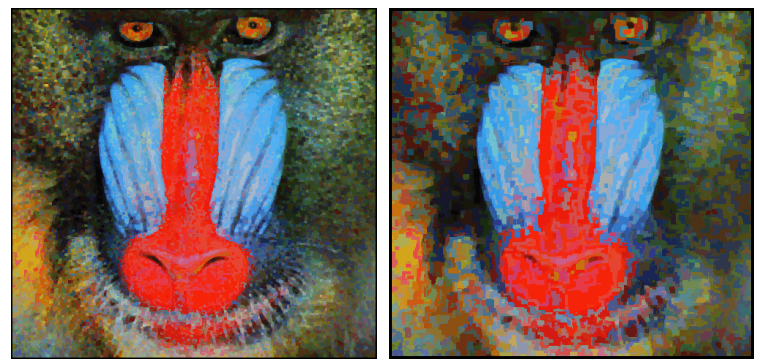

(a) $3 \times 3$ structuring element

(b) $5 \times 5$ structuring element
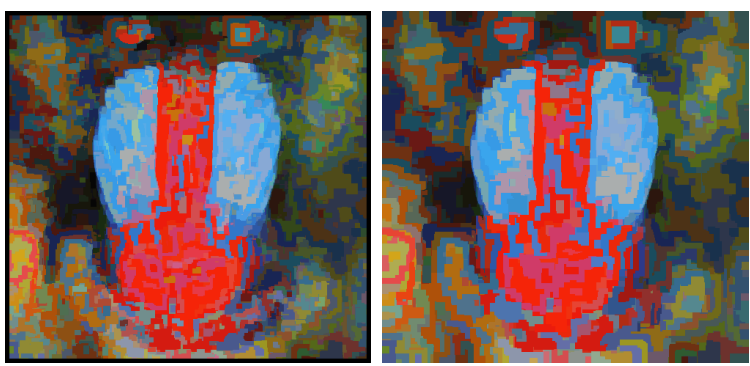

(c) $7 \times 7$ structuring element

\section{Figure 5. Multiscale image representation from openings and closings of increasing scale.}

colour morphological operations. Initial analysis indicates that these operations have potential for use in colour image processing and their application to colour granulometries is an area of ongoing research.

\section{References}

[1] J. Astola, P. Haavisto, and Y. Neuvo. Vector median filters. Proceedings of IEEE, 78:678-689, 1990.

[2] V. Barnett. The ordering of multivariate data. J. Royal Statistical Society A, 139(Part 3):318-343, 1976.

[3] M. Comer and E. Delp. Morphological operations for color image processing. Journal of Electronic Imaging, 8(3):279289, 1999.

[4] A.N. Evans. Vector area morphology for motion field smoothing and interpretation. IEE Proc. Vision, Image and Signal Processing, 150(4):219-226, August 2003.

[5] A.N. Evans. Morphological gradient operators for colour images. In IEEE International Conference on Image Processing, pages Vol III: 3089-3092, 2004.

[6] A.N. Evans and X.U. Liu. A morphological gradient approach to colour edge detection. IEEE Trans. Image Processing, In Press.

[7] S. Gibson, R. Harvey, and G.D. Finlayson. Convex colour sieves. In Proc. 4th International Conference on Scale Space Methods in Computer Vision, volume LNCS 2695, pages 550-563, 2003.

[8] S. Gibson, R. Harvey, and G.D. Finlayson. Evaluating a colour scale-space. In Proc. British Machine Vision Conference, June 2003.

[9] D. Gimenez and A.N. Evans. Colour morphological scalespaces for image segmentation. In Proc. British Machine Vision Conference, pages 909-918, September 2005.

[10] A. Hanbury and J. Serra. Mathematical morphology in the hls colour space. In 12th British Machine Vision Conference, 2001.

[11] J. Li and Y. Li. Multivariate mathematical morphology based on principal component analysis: initial results in building extraction. International Archives for Photogrammetry, 35(B7):1168-1173, 2004.

[12] K.N. Plataniotis, D. Androutsos, and A.N. Venetsanopoulos. Adaptive fuzzy systems for multichannel signal processing. 87(9):1601-1622, 1999.

[13] K.N. Plataniotis and A.N. Venetsanopoulos. Color Image Processing and Applications. Springer Verlag, 2000.

[14] L.J. Sartor and A.R. Weeks. Morphological operations on color images. Journal of Electronic Imaging, 10(2):548$559,2001$.

[15] H. Talbot, C. Evans, and R. Jones. Complete ordering and multivariate mathematical morphology. In H. Heijmans and J. Roerdink, editors, Mathematical Morphology and Its Applications to Image and Signal Processing, pages 27-34, Amsterdam, 1998. Kluwer Academic Press.

[16] P.E. Trahanias and A.N. Venetsanopoulos. Color edge detection using vector order statistics. IEEE Trans. Image Processing, 2(2):259-264, 1993.

[17] P.E. Trahanias and A.N. Venetsanopoulos. Vector directional filters - a new class of multichannel image processing filters. IEEE Trans. Image Processing, 2(4):528-534, 1993.

[18] P.E. Trahanias and A.N. Venetsanopoulos. Vector order statistics operators as color edge detectors. IEEE Trans. Systems, Man and Cybernetics, 26(1):135-143, February 1996.

[19] T. Viero, K. Oistamo, and Y. Neuvo. Three-dimensional median-related filters for colour image sequence filtering. IEEE Trans. Circuits and Systems for Video Technology, 4(2):129-142, 1994. 